\title{
THE PIMSNER-VOICULESCU SEQUENCE FOR COACTIONS OF COMPACT LIE GROUPS
}

\author{
MAGNUS GOFFENG
}

\begin{abstract}
The Pimsner-Voiculescu sequence is generalized to a Pimsner-Voiculescu tower describing the $K K$-category equivariant with respect to coactions of a compact Lie group satisfying the Hodgkin condition. A dual Pimsner-Voiculescu tower is used to show that coactions of a compact HodgkinLie group satisfy the Baum-Connes property.
\end{abstract}

\section{Introduction}

When $G$ is a second countable, locally compact group and $A$ is a separable $C^{*}$ algebra with a continuous $G$-action, the Baum-Connes conjecture states that the $K$-theory of the reduced crossed product $A \rtimes_{r} G$ can be calculated by means of geometric and representation theoretical properties of $G$ and $A$, see more in [4]. To be more precise, the Baum-Connes conjecture states that the assembly mapping $\mu_{A}: K_{*}^{G}(\mathscr{E} G ; A) \rightarrow K_{*}\left(A \rtimes_{r} G\right)$ is an isomorphism. The space $\mathscr{E} G$ is the universal proper $G$-space and $K_{*}^{G}(\mathscr{E} G ; A)$ is the proper equivariant $K$-homology with coefficients in $A$. There are known counterexamples when $\mu_{A}$ is not an isomorphism, so it is more natural to speak of groups having the Baum-Connes property. In [10], the equivariant $K$-homology with coefficients in $A$ was proved to be the left derived functor of $F(A)=K_{*}\left(A \rtimes_{r} G\right)$ and the assembly mapping being the natural transformation from $L F$ to $F$. The approach to the Baum-Connes property using triangulated categories can be generalized to discrete quantum groups, see [9], which indicates that geometric techniques such as universal proper $G$-spaces can be generalized to discrete quantum groups.

The generalization of the Baum-Connes property to quantum groups has been studied in for instance [11] and [17]. The case studied in [11] is that of quantum group actions of the dual of a compact Lie group which correspond to coactions of the Lie group. In [11] duals of compact Lie groups were shown to satisfy the strong Baum-Connes property, i.e., the embedding of

Received 29 October 2010, in final form 4 January 2011. 
the triangulated category generated by proper coactions, the $C^{*}$-algebras that are Baaj-Skandalis dual to trivial $G$-actions, into the $K K$-category equivariant with respect to coactions is essentially surjective. In this paper we construct an analogue of the Pimsner-Voiculescu sequence for coactions of a compact Hodgkin-Lie group $G$ that describes how the $K K$-category equivariant with respect to coactions of $G$ is built up from the $C^{*}$-algebras with coactions of $G$ which are proper in the sense of [11].

The starting point is to express the Pimsner-Voiculescu sequence for Zactions in terms of a property of the representation ring of a rank one torus. Using the Universal Coefficient Theorem, the Pimsner-Voiculescu sequence can be constructed from a Koszul complex

$$
0 \longrightarrow R(T) \stackrel{\alpha}{\longrightarrow} R(T) \longrightarrow 0
$$

where $\alpha$ is defined as multiplication by $1-t$ under the isomorphism $R(T) \cong$ $\mathrm{Z}\left[t, t^{-1}\right]$. When $A$ has a coaction of $T$, i.e., a Z-action, the tensor product over $R(T)$ between this Koszul complex and $K_{*}^{T}\left(A \rtimes_{r} \mathrm{Z}\right)$ gives the PimsnerVoiculescu sequence. In the generalization to higher rank, when $T$ is a torus of rank $n$ we consider the Koszul complex

$$
\begin{aligned}
0 \longrightarrow \wedge^{n} R(T)^{n} \longrightarrow \wedge^{n-1} R(T)^{n} \longrightarrow \cdots & \\
& \longrightarrow \wedge^{2} R(T)^{n} \longrightarrow R(T)^{n} \longrightarrow R(T) \longrightarrow 0 .
\end{aligned}
$$

The boundary mappings in this complex are defined from interior multiplication with the element $\sum\left(1-t_{i}\right) e_{i}^{*} \in \operatorname{Hom}_{R(T)}\left(R(T)^{n}, R(T)\right)$. If $G$ is a compact Hodgkin-Lie group with maximal torus $T$, the representation $\operatorname{ring} R(T)$ is a free $R(G)$-module by [15], so the generalization from a torus to compact Hodgkin-Lie groups goes in a straightforward fashion. Just as when the rank is 1 , the Koszul complex above can be used to produce sequence of distinguished triangles which is the analogue of a Pimsner-Voiculescu sequence for the $K$-theory of crossed products by coactions of $G$.

We will give a geometric description of a sequence of distinguished triangles in the $K K$-category equivariant with respect to coactions of $G$ that corresponds to the above Koszul complex under the Universal Coefficient Theorem. As for the Pimsner-Voiculescu sequence for $Z$ we will obtain a projective resolution of the crossed product by a coaction in the sense of triangulated categories rather than exact sequences. Using suitable tensor products we produce in Theorem 3.4 a sequence of distinguished triangles in the $K K$-category equivariant with respect to coactions of $G$ that we call the generalized Pimsner-Voiculescu 
tower for $A$ :

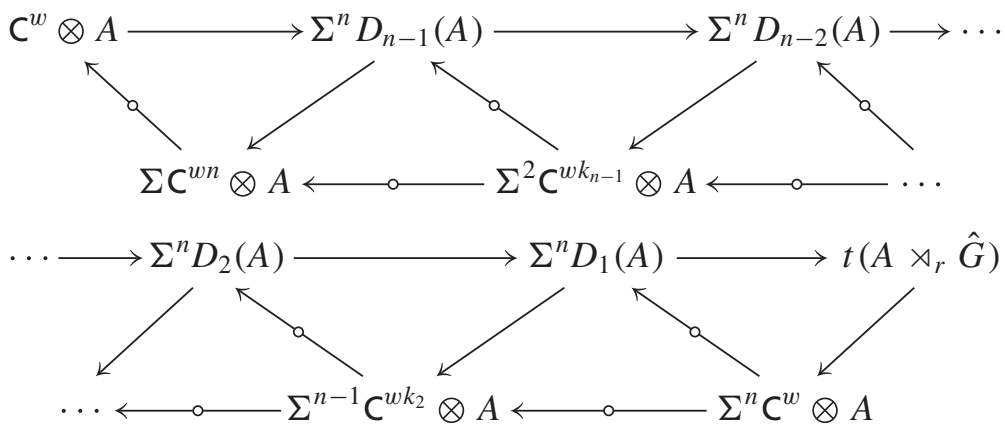

Here $t\left(A \rtimes_{r} \hat{G}\right)$ denotes the $C^{*}$-algebra $A \rtimes_{r} \hat{G}$ equipped with the trivial $\hat{G}$-action and the terms $D_{i}(A)$ can be explicitly described as braided tensor products. Taking $K$-theory of the lower row will give a complex similar to the Koszul complex that in a sense forms a projective resolution of the $K$-theory of $A \rtimes \hat{G}$. The dual Pimsner-Voiculescu gives a more precise description of the results of [11] by a sequence of distinguished triangles in $K K^{G}$ that describes the crossed product $A \rtimes_{r} \hat{G}$ in terms of $G-C^{*}$-algebras with trivial $G$-action, thus giving a direct route to the strong Baum-Connes property of $\hat{G}$.

The paper is organized as follows; the first section consists of a review of $K K$-theory of actions and coactions. In particular we gather some known results about the braided tensor product and the Drinfeld double which plays a mayor role in constructing the dual Pimsner-Voiculescu tower. The main references of this section are [1], [2], [3], [7], [10], [12] and [16]. In the second section a geometric construction of the Pimsner-Voiculescu sequence for Z-actions is presented and generalized to higher rank via a Koszul complex. In the third section the restriction functor for coactions is used to generalize the PimsnerVoiculescu sequence to coactions of compact Hodgkin-Lie groups $G$. As an example of this we calculate the $K$-theory of some compact homogeneous spaces. By similar methods, a dual Pimsner-Voivulescu tower is constructed in $K K^{G}$, following the ideas of [10]. At the end of the paper we discuss some possible generalizations to duals of Woronowicz deformations.

ACKNOWLEDGMENTs: The author would like to thank Ryszard Nest for posing the question on how to explicitly construct the crossed product of a $\hat{G}-$ $C^{*}$-algebra from trivial actions and for much inspiration in the writing process. The author also wishes to thank the referee for several valuable suggestions. 


\section{Actions and coactions of compact groups}

The standard approach to equivariant $K$-theory is to introduce equivariant $K K$ theory. If $A$ and $B$ are two separable $C^{*}$-algebras with a continuous action of a locally compact group $G$, the equivariant $K K$-group $K K^{G}(A, B)$ is defined as the set of homotopy classes of $G$-equivariant $A-B$-Kasparov modules which forms an abelian group under direct sum. The $K K$-groups can be equipped with a product such that if $C$ is a third separable $C^{*}$-algebra with a continuous $G$-action there is an additive pairing called the Kasparov product

$$
K K^{G}(A, B) \times K K^{G}(B, C) \longrightarrow K K^{G}(A, C) .
$$

Following the standard construction, we let $K K^{G}$ denote the additive category of all separable $C^{*}$-algebras with a continuous $G$-action and a morphism in $K K^{G}$ from $A$ to $B$ is an element of $K K^{G}(A, B)$. The composition of two $K K^{G}$ morphisms is defined to be their Kasparov product. The group $K K^{G}(\mathrm{C}, A)$ coincides with the equivariant $K$-theory of $A$. In particular, if $G$ is compact $K K^{G}(\mathrm{C}, \mathrm{C})=R(G)$, the representation ring of $G$. The action of $R(G)$ on equivariant $K$-theory generalizes to an $R(G)$-module structure on the bivariant groups $K K^{G}(A, B)$.

The category $K K^{G}$ can be equipped with a triangulated structure with a mapping cone coming from the mapping cone construction of a $*$-homomorphism. The triangulated structure on $K K^{G}$ is universal in the sense that any homotopy invariant, stable, split-exact functor on the category of $C^{*}$-algebras with a continuous $G$-action defines a homological functor on $K K^{G}$. The construction of the triangulated structure and its universality are thoroughly explained in [10]. Let us just recall the basics of the construction of the triangulated structure on $K K^{G}$. The suspension $\Sigma A$ of a $G-C^{*}$-algebra is defined by $C_{0}(\mathrm{R}) \otimes A$. By Bott periodicity $\Sigma^{2} \cong \mathrm{id}$. A distinguished triangle in $K K^{G}$ is a triangle isomorphic to one of the form

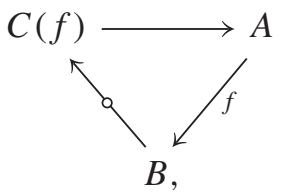

where $C(f)$ is the mapping cone of the equivariant $*$-homomorphism $f$ : $A \rightarrow B$. In particular, if $f: A \rightarrow B$ is a surjection and admits an equivariant completely positive splitting the natural mapping $\operatorname{ker}(f) \rightarrow C(f)$ defines an equivariant $K K$-isomorphism, so under suitable assumptions a distinguished triangle is isomorphic to a short exact sequence.

How to construct $K K$-theory of coactions of groups is easiest seen in the simpler case when $G$ is an abelian group. If $A$ is a $C^{*}$-algebra equipped with an 
action $\alpha$ of the abelian group $G$, the crossed product $A \rtimes_{r} G$ carries a natural action of the Pontryagin dual $\hat{G}$. This action is called the dual action of $\hat{G}$. Since abelian groups are exact, the crossed product by an abelian group defines a triangulated functor $K K^{G} \rightarrow K K^{\hat{G}}$. The crossed product by the dual action is described by Takesaki-Takai duality which states that there is an equivariant isomorphism

$$
A \rtimes_{r} G \rtimes_{r} \hat{G} \cong A \otimes \mathscr{K}\left(L^{2}(G)\right),
$$

where $A \rtimes_{r} G \rtimes_{r} \hat{G}$ is equipped with the dual action of $G$ and the $G$-action on $A \otimes \mathscr{K}\left(L^{2}(G)\right)$ is defined as $\alpha \otimes$ Ad. Takesaki-Takai duality implies that the crossed product defines a triangulated equivalence $K K^{G} \rightarrow K K^{\hat{G}}$.

An action $\alpha$ of a group $G$ on $A$ defines a $*$-homomorphism $\Delta_{\alpha}: A \rightarrow$ $\mathcal{M}\left(A \otimes C_{0}(G)\right)$ by letting $\Delta_{\alpha}(a)$ be the function $g \mapsto \alpha_{g}(a)$. When $G$ is abelian there is a natural isomorphism $C_{0}(\hat{G}) \cong C_{r}^{*}(G)$ and a $\hat{G}$-action corresponds to a non-degenerate $*$-homomorphism $\Delta_{A}: A \rightarrow \mathscr{M}\left(A \otimes_{\min } C_{r}^{*}(G)\right)$ satisfying certain conditions. The first instance of a coaction of a group $G$ is on $C_{r}^{*}(G)$. Using the universal property of $C_{r}^{*}(G)$, one can construct a non-degenerate mapping $\Delta: C_{r}^{*}(G) \rightarrow \mathscr{M}\left(C_{r}^{*}(G) \otimes_{\min } C_{r}^{*}(G)\right)$ called the comultiplication and is induced from the diagonal homomorphism $G \rightarrow G \times G$. Clearly, the mapping $\Delta$ satisfies:

$$
(\Delta \otimes \mathrm{id}) \Delta=(\operatorname{id} \otimes \Delta) \Delta,
$$

so we say that $\Delta$ is coassociative. Since $\Delta_{21}=\Delta$ the comultiplication $\Delta$ is cocommutative, so if we interpret $C_{r}^{*}(G)$ as the functions on a reduced locally compact quantum group $\hat{G}$ then $\hat{G}$ can be thought of as abelian, see more in [7]. With the abelian setting as motivation, we say that a separable $C^{*}$-algebra $A$ has a coaction of the locally compact second countable group $G$ if there is nondegenerate $*$-homomorphism $\Delta_{A}: A \rightarrow \mathcal{M}\left(A \otimes_{\min } C_{r}^{*}(G)\right)$ satisfying the two conditions that $\Delta_{A}(A) \cdot 1_{A} \otimes_{\min } C_{r}^{*}(G)$ is a dense subspace of $A \otimes_{\min } C_{r}^{*}(G)$ and that $\Delta_{A}$ is coassociative in the sense that

$$
\left(\Delta_{A} \otimes \operatorname{id}_{C_{r}^{*}(G)}\right) \Delta_{A}=\left(\operatorname{id}_{A} \otimes \Delta\right) \Delta_{A} .
$$

A separable $C^{*}$-algebra equipped with a coaction of $G$ will be called a $\hat{G}-$ $C^{*}$-algebra. Sometimes we will abuse the notation and call a coaction of $G$ a $\hat{G}$-action. An example of a coaction is the dual coaction on $C^{*}$-algebras of the form $A=B \rtimes_{r} G$, for some $G-C^{*}$-algebra $B$. When $G$ is discrete we can decompose $B \rtimes_{r} G$ by means of the dense subspace $\bigoplus_{g \in G} B \lambda_{g}$ and the dual coaction is defined by $\Delta_{A}\left(b \lambda_{g}\right):=b \lambda_{g} \otimes \lambda_{g}$. In the general setting, the construction of the dual coaction goes analogously and we refer the reader to [1]. 
Much of the theory for group actions also hold for group coactions, the crossed product will as for abelian groups be a stepping stone back and forth between actions and coactions. In [1], the $K K$-theory equivariant with respect to a bi- $C^{*}$-algebras and the corresponding Kasparov product was constructed. In [12] it was proved that the $K K$-theory equivariant with respect to a locally compact quantum group has a triangulated structure defined in the same fashion as for a group.

Let us explain the setting of [1] more explicitly in the case of coactions of a group. An $A-B$-Hilbert bimodule $\mathscr{E}$ is called $\hat{G}$-equivariant if there is a coaction $\delta_{\mathscr{E}}: \mathscr{E} \rightarrow \mathscr{L}_{B \otimes_{\min } C_{r}^{*}(G)}\left(B \otimes_{\min } C_{r}^{*}(G), \mathscr{E} \otimes C_{r}^{*}(G)\right)$ satisfying a coassociativity condition similar to (1) and $\delta_{\mathscr{E}}$ should commute with the $A$-action and $B$-action in the obvious ways. By Proposition 2.4 of [1], the coaction $\delta_{\mathscr{E}}$ is uniquely determined by a unitary $V_{\mathscr{E}} \in \mathscr{L}\left(\mathscr{E} \otimes_{\Delta_{B}}\left(B \otimes_{\min }\right.\right.$ $\left.\left.C_{r}^{*}(G)\right), \mathscr{E} \otimes C_{r}^{*}(G)\right)$ via the equation $\delta_{\mathscr{E}}(x) y=V_{\mathscr{E}}\left(x \otimes_{\Delta_{B}} y\right)$ for $x \in \mathscr{E}$ and $y \in B \otimes_{\min } C_{r}^{*}(G)$. A $\hat{G}$-equivariant $A-B$-Kasparov module is an $A-B$ Kasparov module $(\mathscr{E}, F)$ such that $\mathscr{E}$ is a $\hat{G}$-equivariant $A-B$-Hilbert module and the operator $F$ commutes with the unitary $V_{\mathscr{E}}$ up to a compact operator. The group $K K^{\hat{G}}(A, B)$ is defined as the homotopy classes of $\hat{G}$-equivariant $A-B$ Kasparov modules. The additive category $K K^{\hat{G}}$ is defined by taking the objects to be separable $\hat{G}-C^{*}$-algebras and the group of morphisms from $A$ to $B$ is $K K^{\hat{G}}(A, B)$. The composition in $K K^{\hat{G}}$ is Kasparov product of $\hat{G}$-equivariant Kasparov modules.

To a closed subgroup $H$ of $G$, the restriction of a $G$-action to $H$ defines a restriction functor $\operatorname{Res}_{H}^{G}: K K^{G} \rightarrow K K^{H}$ and its right adjoint is the induction functor $\operatorname{Ind}_{H}^{G}: K K^{H} \rightarrow K K^{G}$. However the restriction goes in the other direction for coactions. When $H$ is a closed subgroup of $G$, there is a non-degenerate embedding $C^{*}(H) \subseteq \mathcal{M}\left(C^{*}(G)\right)$ so a coaction of $H$ can be restricted to a coaction of $G$. This construction defines a triangulated functor $\operatorname{Res}_{\hat{G}}^{\hat{H}}: K K^{\hat{H}} \rightarrow K K^{\hat{G}}$.

The crossed product $B \mapsto B \rtimes_{r} G$ sends a $G-C^{*}$-algebra to a $\hat{G}-C^{*}$ algebra and if $G$ is exact the crossed product induces a triangulated functor $K K^{G} \rightarrow K K^{\hat{G}}$. In order to construct a duality similar to Takesaki-Takai duality one introduces the crossed product by a coaction. If $A$ is a $\hat{G}-C^{*}$-algebra we define

$$
A \rtimes_{r} \hat{G}:=\left[\Delta_{A}(A) \cdot 1_{A} \otimes C_{0}(G)\right] \subseteq \mathscr{M}\left(A \otimes \mathscr{K}\left(L^{2}(G)\right)\right) .
$$

It follows from Lemma 7.2 of [2] that $A \rtimes_{r} \hat{G}$ forms a $C^{*}$-algebra. For a thorough introduction to crossed products by coactions see [13]. The $C^{*}$ algebra $A \rtimes_{r} \hat{G}$ carries a continuous $G$-action defined in the dense subspace 
$\Delta_{A}(A) \cdot 1_{A} \otimes C_{0}(G)$ by

$$
\text { g. }\left(\Delta_{A}(a) \cdot 1_{A} \otimes f\right):=\Delta_{A}(a) \cdot 1_{A} \otimes g . f .
$$

Similarly to the abelian setting, Takesaki-Takai duality holds so there are equivariant isomorphisms $B \rtimes_{r} G \rtimes_{r} \hat{G} \cong B \otimes \mathscr{K}\left(L^{2}(G)\right)$ and $A \rtimes_{r} \hat{G} \rtimes_{r} G \cong$ $A \otimes \mathscr{K}\left(L^{2}(G)\right)$ which ensures that the crossed product defines an equivalence of triangulated categories known as Baaj-Skandalis duality.

The tensor product on the category of $G-C^{*}$-algebras is well defined. If $A$ and $B$ have actions $\alpha$ respectively $\beta$ of $G$ the tensor product $A \otimes_{\min } B$ can be equipped with the action $\alpha \otimes \beta: G \rightarrow \operatorname{Aut}\left(A \otimes_{\min } B\right)$. However, for a non-abelian group $G$ the construction of a tensor product of $\hat{G}-C^{*}$-algebras can not be done by just taking tensor products of the $C^{*}$-algebras. The tensor product relevant for $\hat{G}-C^{*}$-algebras is the braided tensor product over $\hat{G}$ which requires one further structure. Suppose that $A$ is a $\hat{G}$-algebra with a continuous $G$-action $\alpha$. If the action $\alpha$ satisfies that

$$
\Delta_{A} \circ \alpha_{g}=\left(\alpha_{g} \otimes \operatorname{Ad}(g)\right) \Delta_{A}
$$

we say that $A$ is a Yetter-Drinfeld algebra. An example of a Yetter-Drinfeld algebra is $C_{r}^{*}(G)$ with $G$-action defined by the adjoint action $G \rightarrow \operatorname{Aut}(G)$. It is much easier to construct a Yetter-Drinfeld algebra from a $G-C^{*}$-algebra, if $A$ is a $G-C^{*}$-algebra we can in a functorial way define a coaction of $G$ on $A$ by setting $\Delta_{A}(a):=a \otimes 1$. When $A$ is a Yetter-Drinfeld algebra, the $C^{*}$-algebra $A \rtimes_{r} \hat{G}$ is also a Yetter-Drinfeld algebra since the morphism $\Delta_{A}$ is covariant with respect to the $G$-action and $\Delta_{A}$ extends to a coaction of $G$ on $A \rtimes_{r} \hat{G}$, see more in [12]. This construction is functorial and the crossed product can be seen as a functor on the category of Yetter-Drinfeld algebras.

When $A$ is a Yetter-Drinfeld algebra and $B$ is a $\hat{G}-C^{*}$-algebra we define the mappings

$$
\begin{array}{ll}
\iota_{A}: A \longrightarrow \mathscr{M}\left(A \otimes_{\min } B \otimes \mathscr{K}\left(L^{2}(G)\right)\right), & \iota(a):=\Delta_{\alpha}(a)_{13} \\
\iota_{B}: B \longrightarrow \mathscr{M}\left(A \otimes_{\min } B \otimes \mathscr{K}\left(L^{2}(G)\right)\right), & \iota(b):=\Delta_{B}(b)_{23} .
\end{array}
$$

Following [12], the braided tensor product $A \bigotimes_{\hat{G}} B$ is defined as the closed linear span of $\iota_{A}(A) \cdot \iota_{B}(B)$. By Proposition 8.3 of [16], $A \nabla_{\hat{G}} B$ forms a *-subalgebra of $\mathscr{M}\left(A \otimes_{\min } B \otimes \mathscr{K}\left(L^{2}(G)\right)\right)$ so the braided tensor product is a $C^{*}$-algebra. The coaction of $G$ on $A \bigotimes_{\hat{G}} B$ is defined by

$$
\Delta_{A} \otimes_{\hat{G}} \Delta_{B}\left(\iota_{A}(a) \cdot \iota_{B}(b)\right):=\left(\iota_{A} \otimes \mathrm{id}\right)\left(\Delta_{A}(a)\right) \cdot\left(\iota_{B} \otimes \mathrm{id}\right)\left(\Delta_{B}(b)\right) .
$$

Observe that since $C_{r}^{*}(G)$ is cocommutative, the adjoint $\hat{G}$-action is trivial and a similar construction of a braided tensor product over $G$ between $G-$ 
$C^{*}$-algebras with trivial $\hat{G}$-actions coincides with the usual tensor product. In general, the braided tensor product over $G$ does not need to coincide with the usual tensor product. By Lemma 3.5 of [12] there is a $G$-equivariant isomorphism

$$
\left(A \bigotimes_{\hat{G}} B\right) \rtimes_{r} \hat{G} \cong\left(A \rtimes_{r} \hat{G}\right) \bigotimes_{\hat{G}} B
$$

where the $G$-coaction on the right hand side is the trivial one on $B$. More generally, this identity holds for any quantum group and in particular also for braided tensor products over $G$. We will prove this statement in special case of braided tensor products over a compact group $G$ with $C(G)$ below in Lemma 3.3.

If we interpret the structure of a Yetter-Drinfeld algebra as two actions of the quantum groups $G$ and $\hat{G}$ satisfying a certain cocycle relation, the cocycle defines a quantum group by means of a double crossed product such that Yetter-Drinfeld algebras are precisely the $C^{*}$-algebras with an action of this double crossed product. The right quantum group to look at is the Drinfeld double $D(G)$. Using the notations of quantum groups, the algebra of functions on $D(G)$ is $C_{0}\left(G, C_{r}^{*}(G)\right)=C_{0}(G) \otimes C_{r}^{*}(G)$ with the obvious action and coaction of $G$. The action and coaction define a comultiplication

$$
\Delta_{D(G)}: C_{0}(D(G)) \longrightarrow \mathscr{M}\left(C_{0}(D(G)) \otimes C_{0}(D(G))\right)
$$

by $\Delta_{D(G)}:=\sigma_{23} \operatorname{Ad}\left(W_{23}\right)\left(\Delta_{C_{0}(G)} \otimes \Delta_{C_{r}^{*}(G)}\right)$ where $W \in \mathscr{B}\left(L^{2}(G) \otimes L^{2}(G)\right)$ is the multiplicative unitary of $G$ defined by $W f\left(g_{1}, g_{2}\right)=f\left(g_{1}, g_{1} g_{2}\right)$. The comultiplication $\Delta_{D(G)}$ makes $D(G)$ into a quantum group by Theorem 5.3 of [3]. A Yetter-Drinfeld algebra $A$ with the action $\alpha$ and coaction $\Delta_{A}$ correspond to a $D(G)-C^{*}$-algebra by defining the $D(G)$-coaction

$$
\Delta_{A}^{D(G)}:=\left(\Delta_{\alpha} \otimes \mathrm{id}\right) \Delta_{A}: A \longrightarrow \mathcal{M}\left(A \otimes_{\min } C_{0}(D(G))\right),
$$

see more in Proposition 3.2 of [12]. Therefore we can consider the braided tensor product as a tensor product between $D(G)-C^{*}$-algebras and $\hat{G}-C^{*}$ algebras. The braided tensor product induces a biadditive functor

$$
\otimes_{\hat{G}}: K K^{D(G)} \times K K^{\hat{G}} \longrightarrow K K^{\hat{G}} .
$$

Much of the theory of coactions can be done without introducing any quantum groups, but in order to construct the Pimsner-Voiculescu sequence for coactions of compact Hodgkin-Lie groups we will need the braided tensor product as a biadditive functor between $K K$-categories. 


\section{The Pimsner-Voiculescu sequence from the viewpoint of representation rings}

In this section we will study the Pimsner-Voiculescu sequence for $Z$ and generalize to a Pimsner-Voiculescu tower for $Z^{n}$. We will use representation theory to calculate all the mappings explicitly. These calculations will in a surprisingly straightforward way give a natural route to a Pimsner-Voiculescu tower for coactions of compact Lie groups.

Consider the evaluation mapping $l: C_{0}(\mathrm{R}) \rightarrow C_{0}(\mathrm{Z})$. This mapping fits into a $Z$-equivariant short exact sequence

$$
0 \longrightarrow \Sigma C_{0}(\mathrm{Z}) \longrightarrow C_{0}(\mathrm{R}) \stackrel{l}{\longrightarrow} C_{0}(\mathrm{Z}) \longrightarrow 0 .
$$

The Z-equivariant Dirac operator $\not D$ on $R$ defines a Z-equivariant odd unbounded $K$-homology class, thus an element $[\not D] \in K K^{\mathrm{Z}}\left(C_{0}(\mathrm{R}), \Sigma \mathrm{C}\right)$. While $\mathrm{R}$ is the universal proper $Z$-space the element $[D]$ is the Dirac element of $Z$ and the strong Baum-Connes property of $Z$ implies that $[\not D]$ is a $K K^{Z}$-isomorphism. The exact sequence (4) induces a distinguished triangle in $K K^{Z}$ which after using the isomorphism $C_{0}(\mathrm{R}) \cong \Sigma \mathrm{C}$ and rotation 4 steps to the left becomes

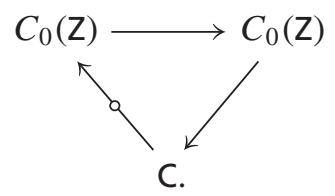

In a certain sense, the distinguished triangle (5) captures the entire behavior of the Pimsner-Voiculescu sequence. If $A$ is a $Z-C^{*}$-algebra we can apply BaajSkandalis duality to (5) and tensor with $A \rtimes_{r}$ Z. If we apply Baaj-Skandalis duality again, we obtain a distinguished triangle in $K K^{Z}$ :

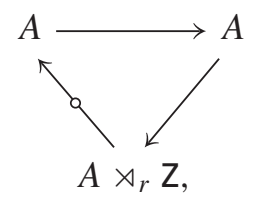

where $A \rtimes_{r} \mathrm{Z}$ is given the trivial Z-action. Taking $K$-theory of this distinguished triangle gives back the classical Pimsner-Voiculescu sequence due to the following proposition:

Proposition 2.1. When $T$ is a torus of rank 1 and the element $\kappa \in$ $K K^{T}(\mathrm{C}, \mathrm{C})$ is defined using the isomorphisms $K K^{T}(\mathrm{C}, \mathrm{C}) \cong \operatorname{Hom}_{R(T)}(R(T)$, $R(T))$ and $R(T) \cong \mathrm{Z}\left[t, t^{-1}\right]$ as

$$
\kappa f\left(t, t^{-1}\right)=(1-t) f\left(t, t^{-1}\right),
$$


the KK-morphism $\kappa$ is Baaj-Skandalis dual to the KK-morphism $C_{0}(\mathrm{Z}) \rightarrow$ $C_{0}(\mathrm{Z})$ defined by (4).

Observe that the $K$-theory of the exact sequence (4) is described from the exact sequence:

$$
0 \longrightarrow R(T) \stackrel{1-t}{\longrightarrow} R(T) \longrightarrow \mathrm{Z} \longrightarrow 0,
$$

by Proposition 2.1. The first terms in this exact sequence is the Koszul complex defined by $1-t \in \operatorname{Hom}_{R(T)}(R(T), R(T))$ and $\mathrm{Z}$ is the cohomology of the Koszul complex.

Proof. Let $\kappa_{0} \in \operatorname{Hom}_{R(T)}(R(T), R(T))$ denote the Baaj-Skandalis dual of the $K K$-morphism induced from (4). It follows directly from the construction that the mapping $R(T) \rightarrow \mathrm{Z}$ induced from $\Sigma C_{0}(\mathrm{Z}) \rightarrow C_{0}(\mathrm{R})$ is the augmentation mapping $\mathrm{Z}\left[t, t^{-1}\right] \rightarrow \mathrm{Z}$ onto the generator of $K_{1}\left(C_{0}(\mathrm{R})\right)$. Therefore the image of $\kappa_{0}$ is the ideal generated by either $1+t$ of $1-t$ so $\kappa_{0}$ is of the form $u \cdot(1 \pm t)$ for some unit $u \in \mathrm{Z}\left[t, t^{-1}\right]$. The sign and $u=1$ is found by either a direct calculation or by considering the Pimsner-Voiculescu sequence for $C_{0}(\mathrm{Z})$.

We will return to the Koszul complexes later on. First we will construct a geometric interpretation of the higher rank situation. Assume that $T$ is a torus of rank $n$ and consider the semi-open unit cube $I=\left[0,1{ }^{n} \subseteq \mathrm{R}^{n}\right.$. For $i=1, \ldots, n$ we define $\tilde{X}_{i}$ as the set of open $i-1$-dimensional faces of $I$. The union satisfies

$$
\bigcup_{i=1}^{n} \tilde{X}_{i}=\partial I \cap I .
$$

We let $k_{i}$, for $i=1,2, \ldots n$, denote the integers $k_{i}:=\left(\begin{array}{c}n \\ i-1\end{array}\right)$. The set $\tilde{X}_{i}$ has $k_{i}$ connected components so if we choose a homeomorphism ]0, $1[\cong \mathrm{R}$ there are homeomorphisms

$$
\tilde{X}_{i} \cong \coprod_{j=1}^{k_{i}} \mathrm{R}^{i-1} \quad \text { for } \quad i=1,2, \ldots, n,
$$

where we interpret $\mathrm{R}^{0}$ as the one-point space. We take $X_{i}$ to be the $Z^{n}$-translates of $\bigcup_{j \leq i} \tilde{X}_{j}$ and define $Y_{i}:=\mathrm{R}^{n} \backslash X_{i}$ for $1=1,2, \ldots, n$ and $Y_{0}:=\mathrm{R}^{n}$.

Proposition 2.2. For $i=1,2, \ldots, n$ there are $\mathrm{Z}^{n}$-equivariant isomorphisms

$$
C_{0}\left(Y_{i-1}\right) / C_{0}\left(Y_{i}\right) \cong \mathrm{C}^{k_{i}} \otimes \Sigma^{i-1} C_{0}\left(\mathrm{Z}^{n}\right) .
$$


Proof. By equation (6) there is a $Z^{n}$-equivariant homeomorphism

$$
Y_{i-1} \backslash Y_{i} \cong \coprod_{m \in Z^{n}}\left(\coprod_{j=1}^{k_{i}} \mathrm{R}^{i-1}\right),
$$

where $Z^{n}$ acts by translation on the first disjoint union. Therefore

$$
\begin{aligned}
C_{0}\left(Y_{i-1}\right) / C_{0}\left(Y_{i}\right) & \cong C_{0}\left(Y_{i-1} \backslash Y_{i}\right) \cong C_{0}\left(\coprod_{m \in \mathrm{Z}^{n}}\left(\coprod_{j=1}^{k_{i}} \mathrm{R}^{i-1}\right)\right) \\
& \cong \mathrm{C}^{k_{i}} \otimes C_{0}\left(\mathrm{Z}^{n} \times \mathrm{R}^{i-1}\right) \equiv \mathrm{C}^{k_{i}} \otimes \Sigma^{i-1} C_{0}\left(\mathrm{Z}^{n}\right) .
\end{aligned}
$$

Consider the classifying space $\mathrm{R}^{n}$ for proper actions of $\mathrm{Z}^{n}$. Since $\mathrm{Z}^{n}$ has the strong Baum-Connes property, the Dirac element $[\not D]$ induces a $K K^{Z^{n}}$ isomorphism $C_{0}\left(\mathrm{R}^{n}\right) \cong \Sigma^{n} \mathrm{C}$. An alternative approach to constructing this isomorphism is the Julg theorem which implies that for any $T-C^{*}$-algebra $A$ there is an isomorphism $K_{*}^{T}(A) \cong K_{*}\left(A \rtimes_{r} T\right)$. Therefore $K_{*}^{T}\left(\Sigma^{n} \mathrm{C} \rtimes \mathrm{Z}^{n}\right) \cong$ $K_{*}^{T}\left(C_{0}\left(\mathrm{R}^{n}\right) \rtimes \mathrm{Z}^{n}\right)$ and the statement follows from the Universal Coefficient Theorem for the compact Hodgkin-Lie group $T$, see more in [14].

For $i=1,2, \ldots, n$, Proposition 2.2 implies that there is a $Z^{n}$-equivariant short exact sequence

$$
0 \longrightarrow C_{0}\left(Y_{i}\right) \longrightarrow C_{0}\left(Y_{i-1}\right) \longrightarrow \mathrm{C}^{k_{i}} \otimes \Sigma^{i-1} C_{0}\left(\mathrm{Z}^{n}\right) \longrightarrow 0 .
$$

We will by $\kappa_{i} \in K K^{Z^{n}}\left(\mathrm{C}^{k_{i}} \otimes C_{0}\left(\mathrm{Z}^{n}\right), \mathrm{C}^{k_{i+1}} \otimes C_{0}\left(\mathrm{Z}^{n}\right)\right)$ denote the $\mathrm{Z}^{n}$-equivariant $K K$-morphism defined in such a way that the extension class defined by (7) composed with the restriction mapping $C_{0}\left(Y_{i}\right) \rightarrow \mathrm{C}^{k_{i+1}} \otimes \Sigma^{i} C_{0}\left(\mathrm{Z}^{n}\right)$ coincides with $\Sigma^{i-1} \kappa_{i}$. Notice that $\left.Y_{n}=Z^{n} \times\right] 0,1\left[^{n}\right.$ and $Y_{0}=\mathrm{R}^{n}$ so we have that $C_{0}\left(Y_{n}\right)=\Sigma^{n} C_{0}\left(\mathrm{Z}^{n}\right)$ and $C_{0}\left(Y_{0}\right)=C_{0}\left(\mathrm{R}^{n}\right)$, the latter being $K K^{\mathrm{Z}^{n}}$-isomorphic to $\Sigma^{n} C$. Thus we get a sequence of distinguished triangles in $K K^{Z^{n}}$ :

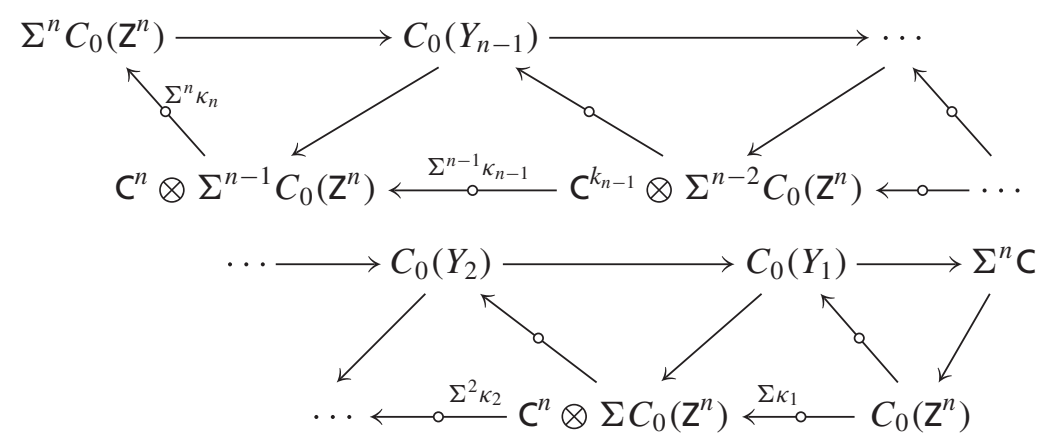


A sequence of distinguished triangles of this type will be called a tower. The tower (8) in $K K^{2^{n}}$ is the higher rank analogue of the distinguished triangle (5). The tower (8) can be generalized to contain any coefficient ring.

To find a better description of the morphisms $\kappa_{i}$ let us recall the notion of a Koszul complex. Let $R$ denote a commutative ring and $E$ an $R$-module. For simplicity we will assume that $E$ is free and finitely generated, let us say of rank $N$. For an element $v \in \operatorname{Hom}_{R}(E, R)$, the Koszul complex of $E$ with respect to $v$ is the complex

$$
0 \longrightarrow \wedge^{N} E \stackrel{\partial_{1}}{\longrightarrow} \wedge^{N-1} E \stackrel{\partial_{2}}{\longrightarrow} \cdots \stackrel{\partial_{N-2}}{\longrightarrow} \wedge^{2} E \stackrel{\partial_{N-1}}{\longrightarrow} E \stackrel{v}{\longrightarrow} R \longrightarrow 0,
$$

where each $\partial_{k}$ is defined as interior multiplication by $v$. Since we have assumed $E$ to be free, we may write $v=\sum v_{i} \mathrm{e}_{i}^{*}$ for some $v_{1}, v_{2}, \ldots, v_{N} \in R$ and the dual basis $\mathrm{e}_{i}^{*}$ of a basis $\mathrm{e}_{i}, i=1,2, \ldots, N$ of $E$. If the sequence $v_{1}, v_{2}, \ldots, v_{N}$ is a regular sequence the Koszul complex is exact except at $R$. The cohomology of the Koszul complex is in this case $R / v(E)$ at $R$. See more in [5].

The Koszul complex of interest to us is constructed from the module $E:=$ $R(T)^{n}$ over the representation ring of the torus $T$ which has the following form:

$$
R(T) \cong \mathrm{Z}\left[t_{1}^{ \pm 1}, \ldots, t_{n}^{ \pm 1}\right] .
$$

Observe that Baaj-Skandalis duality and the Universal Coefficient Theorem implies that

$$
\begin{aligned}
K K^{\mathrm{Z}^{n}}\left(\mathrm{C}^{k_{i}} \otimes C_{0}\left(\mathrm{Z}^{n}\right), \mathrm{C}^{k_{i+1}} \otimes C_{0}\left(\mathrm{Z}^{n}\right)\right) & \cong K K^{T}\left(\mathrm{C}^{k_{i}}, \mathrm{C}^{k_{i+1}}\right) \\
& \cong \operatorname{Hom}_{R(T)}\left(R(T)^{k_{i}}, R(T)^{k_{i+1}}\right) .
\end{aligned}
$$

We have that $R(T)^{k_{i}} \cong \wedge^{n-i+1} E$ so the lower row in (8) have the right ranks for coinciding with a Koszul complex. Let $f_{i} \in \operatorname{Hom}_{R(T)}\left(\wedge^{n-i+1} E, \wedge^{n-i} E\right)$ denote the image of $\kappa_{i}$ under the isomorphisms above. To simplify notations, we will by $\left(e_{i}\right)_{i=1}^{n}$ denote the $R(T)$-basis of $E$ coming from the isomorphism $E \cong R(T) \otimes_{\mathrm{Z}} \mathrm{Z}^{n}$ and by $\left(e_{i}^{*}\right)_{i=1}^{n}$ denote the dual basis.

THEOREM 2.3. Under the isomorphisms $R(T)^{k_{i}} \cong \wedge^{n-i+1}$ E the mappings $f_{i}$ coincide with interior multiplication by the element $v:=\sum_{i=1}^{n}\left(1-t_{i}\right) \mathrm{e}_{i}^{*}$. Therefore the sequence

$$
0 \longrightarrow \wedge^{n} E \stackrel{f_{1}}{\longrightarrow} \wedge^{n-1} E \stackrel{f_{2}}{\longrightarrow} \cdots \stackrel{f_{n-2}}{\longrightarrow} \wedge^{2} E \stackrel{f_{n-1}}{\longrightarrow} E \stackrel{f_{n}}{\longrightarrow} R(T) \longrightarrow 0
$$

defines a complex isomorphic to the Koszul complex of E whose cohomology at $R(T)$ is Z. 
Proof. While both $f_{i}$ and the mapping defined by interior multiplication by $v$ are $R(T)$-linear it is sufficient to prove that $f_{i}(u)=v \neg u$ for elements of the form $u=\mathrm{e}_{m_{1}} \wedge \cdots \wedge \mathrm{e}_{m_{n-i+1}} \in \wedge^{n-i+1} E$, where $m_{1}, \ldots, m_{n-i+1} \in$ $\{1,2, \ldots, n\}$. Let $\left(m_{p}\right)_{p=n-i+1}^{n}$ be an enumeration of all $j=1,2, \ldots, n$ such that $j \notin\left(m_{p}\right)_{p=1}^{n-i+1}$. If we view $\mathrm{Z}^{n}$ as a subset of $\mathrm{R}^{n}$ we can define $X_{u} \subseteq \tilde{X}_{i}$ as the open face in $\mathrm{R}^{n}$ spanned by the vectors $\mathrm{e}_{m_{n-i+1}}, \mathrm{e}_{m_{n-i+2}}, \ldots, \mathrm{e}_{m_{n}}$.

Under the isomorphism $\wedge^{n-i+1} E \cong K_{i-1}\left(\mathrm{C}^{k_{i}} \otimes \Sigma^{i-1} C_{0}\left(\mathrm{Z}^{n}\right)\right)$ the element $u$ corresponds to a $K$-theory class on $\tilde{X}_{i}$ which is trivial except on the face $X_{u}$. Therefore there exists sequences of numbers $\left(a_{j}\right)_{j=1}^{n-i+1},\left(b_{j}\right)_{j=1}^{n-i+1} \subseteq \mathrm{Z}$ such that

$$
f_{i}(u)=\sum_{j=1}^{n-i+1}\left(a_{j}+b_{j} t_{j}\right) \mathrm{e}_{m_{j}} \neg u .
$$

If $j=1,2, \ldots, n-i+1$, we will let $X_{u, j}$ denote the open face spanned by the vectors $\mathrm{e}_{m_{j}}, \mathrm{e}_{m_{n-i+1}}, \mathrm{e}_{m_{n-i+2}}, \ldots, \mathrm{e}_{m_{n}}$. It follows from restricting to $X_{u, j}$ that $a_{j}=1$ since Bott periodicity implies that the index mapping $K_{i-1}\left(C_{0}\left(X_{u}\right)\right) \rightarrow$ $K_{i}\left(C_{0}\left(X_{u, j}\right)\right)$ is an isomorphism. In a similar fashion it follows that $b_{i}=-1$.

While $v(E)$ is the ideal generated by the regular sequence $1-t_{1}, 1-$ $t_{2}, \ldots, 1-t_{n}$, the cohomology of the Koszul complex is $R(T) / v(E)=\mathrm{Z}$ and the quotient mapping $R(T) \rightarrow \mathrm{Z}$ coincides with the augmentation mapping.

Consider the tower Baaj-Skandalis dual to (8). Given $A, B \in K K^{T}$ we can apply the homological functor $K K^{T}\left(A,-\otimes_{\min } B\right)$ to this tower. This functor is only homological on the bootstrap category if $B$ is not exact, but all objects in the tower Baaj-Skandalis dual to (8) are in the bootstrap category. The lowest row of the corresponding tower in the category of $R(T)$-modules is a Koszul complex:

$$
\begin{aligned}
0 \longrightarrow \wedge^{n} Z^{n} \otimes K K_{*}^{T}(A, B) \stackrel{\left.v_{A}\right\urcorner}{\longrightarrow} & \wedge^{n-1} Z^{n} \otimes K K_{*}^{T}(A, B) \stackrel{v_{A} \neg}{\longrightarrow} \ldots \\
& \stackrel{\left.v_{A}\right\urcorner}{\longrightarrow} Z^{n} \otimes K K_{*}^{T}(A, B) \stackrel{\left.v_{A}\right\urcorner}{\longrightarrow} K K_{*}^{T}(A, B) \longrightarrow 0
\end{aligned}
$$

where

$$
v_{A}:=\sum_{i=1}^{n}\left(1-\beta_{i *}\right) \mathrm{e}_{i}^{*} \in \operatorname{Hom}_{R(T)}\left(K K_{*}^{T}(A, B)^{n}, K K_{*}^{T}(A, B)\right)
$$

and $\left(\beta_{i}\right)_{i=1}^{n}$ are the commuting equivariant automorphisms of $A$ that are BaajSkandalis to the $Z^{n}$-action on $B \rtimes_{r} T$. The cohomology of this Koszul complex can be calculated from $K K_{*}^{T}(A, B)$. We will return to this subject in the next section in the more general case of Hodgkin-Lie groups and explain this procedure further. 


\section{The generalized Pimsner-Voiculescu-towers}

As mentioned in the introduction, the representation ring $R(T)$ is free over $R(G)$ when $G$ is a Hodgkin-Lie group, so the step to coactions of a compact Hodgkin-Lie group will not be too large. We will throughout this section assume that $G$ is a compact Hodgkin-Lie group of rank $n$ with maximal torus $T$. Recall that a group satisfies the Hodgkin condition if it is connected and the fundamental group is torsion-free.

The embedding $T \subseteq G$ induces a restriction functor $K K^{\hat{T}} \rightarrow K K^{\hat{G}}$. Using the isomorphism $\hat{T} \cong \mathrm{Z}^{n}$, the tower (8) can be restricted to a $K K^{\hat{G}}$-tower:

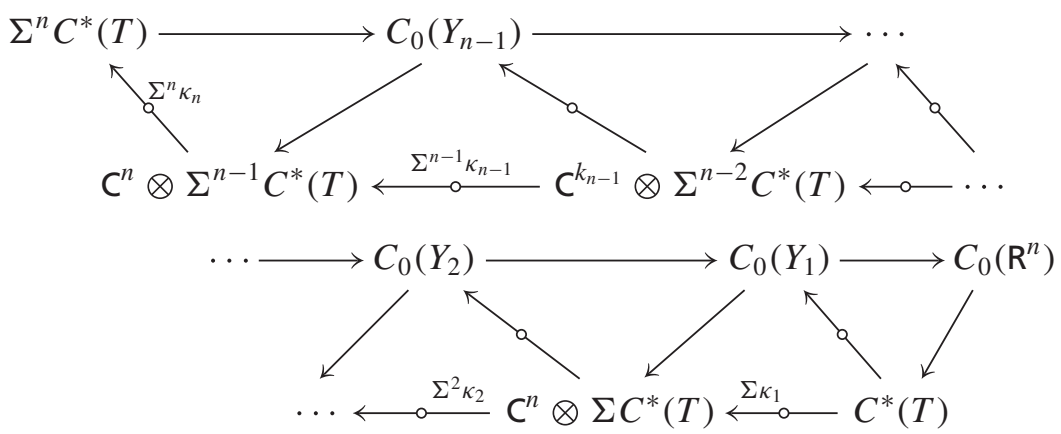

In order to work with this $K K^{\hat{G}}$-tower we need to describe the terms $C^{*}(T)$ in the second row.

LEMMA 3.1. If $G$ is a compact Hodgkin-Lie group with Weyl group of order $w$ there is an isomorphism

$$
C^{*}(T) \cong C^{w} \otimes C^{*}(G) \quad \text { in } K K^{\hat{G}} .
$$

Observe that the condition on $G$ to be a Hodgkin group is equivalent to $\hat{G}$ being a torsion-free quantum group in the sense of Meyer, see [9]. The torsionfree quantum groups are the only non-classical discrete quantum groups for which there is a general formulation of the Baum-Connes property in terms of triangulated categories. In [11], coactions of compact non-Hodgkin Lie groups were considered and the "torsion" turned out to be the torsion elements of $H^{2}\left(G, S^{1}\right)$. The less precise statement $C(G / T) \cong \mathrm{C}^{k}$ in $K K^{G}$ for some $k$ is stated and proved in [11]. An explicit calculation that $k=|W|$ can be found in [15]. We will review the conceptually important part of the proof of a Proposition in [11] which proves Lemma 3.1 aside from the calculation of $k$.

Proof. By [15], the representation ring $R(T)$ is free of rank $w$ over the representation ring $R(G)$ if $\pi_{1}(G)$ is torsion-free. If we let $\mathscr{S}$ denote the 
localizing subcategory of $K K^{G}$ generated by $C$ and $C(G / T)$, Lemma 11 of [10] states that for $A \in \mathscr{S}$ the natural homomorphism

$$
R(T) \otimes_{R(G)} K K^{G}(A, \mathrm{C}) \longrightarrow K K^{T}(A, \mathrm{C})
$$

is an isomorphism. Thus the representable functor on $\mathscr{S}$

$$
A \longrightarrow K K^{G}\left(A, \mathrm{C}^{w}\right) \cong R(T) \otimes_{R(G)} K K^{G}(A, \mathrm{C})
$$

coincides with the representable functor

$$
A \longrightarrow K K^{G}(A, C(G / T)) \cong K K^{T}(A, C) .
$$

The last isomorphism exists as a consequence of the fact that the induction functor $\operatorname{Ind}_{T}^{G}$ is the right adjoint of the restriction functor from $G$ to $T$. So the Yoneda lemma implies that $C(G / T) \cong \mathrm{C}^{w}$ in $\mathscr{S}$ and therefore in $K K^{G}$. Applying Baaj-Skandalis duality it follows that there is an equivariant $K K$ isomorphism $C^{*}(T) \cong \mathrm{C}^{w} \otimes C^{*}(G)$.

Using Lemma 3.1 the tower (8) takes the form:

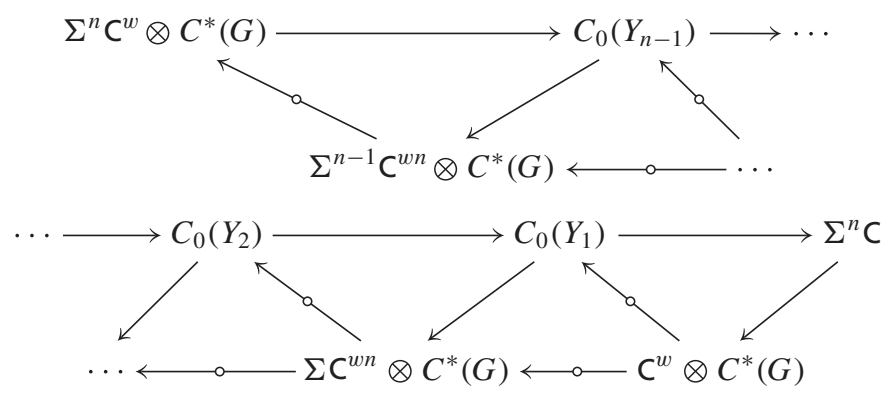

We will call this $K K^{\hat{G}}$-tower the fundamental $G-\mathrm{PV}$-tower. The dual fundamental $G$-PV-tower is defined to be the $K K^{G}$-tower which is Baaj-Skandalis dual to the fundamental $G-\mathrm{PV}$-tower:

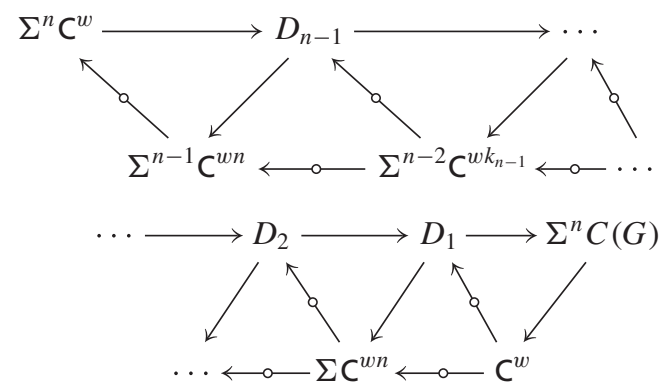

where $D_{i}:=C_{0}\left(Y_{i}\right) \rtimes_{r} \hat{G}$. 
As mentioned above, if $A$ is a $G-C^{*}$-algebra, the trivial coaction of $G$ on $A$ makes $A$ into a Yetter-Drinfeld algebra. This follows from that $C(G)$ is commutative so we can extend a $G$-action via the $D(G)$-equivariant $*$ monomorphism $C(G) \rightarrow \mathscr{M}\left(C_{0}(D(G))\right)$. Clearly, a $G$-equivariant mapping is equivariant in this new $D(G)$-action. Furthermore, since mapping cones does not depend on the action, the trivial extension of a $G$-action to a $D(G)$ action is functorial and respects mapping cones. The following proposition follows from universality.

PROPOSITION 3.2. If $G$ is a locally compact group, the functor mapping a $G-C^{*}$-algebra to a $G$-Yetter-Drinfeld algebra with trivial $\hat{G}$-action defines a triangulated functor $K K^{G} \rightarrow K K^{D(G)}$.

Using the triangulated functor of Proposition 3.2, we may consider the tower (11) as a tower in $K K^{D(G)}$. Applying a crossed product by $G$ we obtain that also the tower (10) is a tower in $K K^{D(G)}$. For a $C^{*}$-algebra $B$ we will use the notation $t(B)$ for the $\hat{G}-C^{*}$-algebra with trivial coaction, or in the context of $G-C^{*}$-algebras $t(B)$ will denote the $G-C^{*}$-algebra with trivial action. Let us state and prove the corresponding version of (3) in a simple case of a braided tensor product over $G$ with $C(G)$, a more general proof can be found in [12].

Lemma 3.3. When B has a continuous $G$-action, there is a $\hat{G}$-equivariant Morita equivalence

$$
(C(G) \otimes B) \rtimes_{r} G \sim_{M} t(B) .
$$

Proof. By Baaj-Skandalis duality, it suffices to prove that there is a $\hat{G}$ equivariant isomorphism $(C(G) \otimes B) \rtimes_{r} G \cong\left(C(G) \rtimes_{r} G\right) \otimes t(B)$. Denote the $G$-action on $B$ by $\beta$ and define the equivariant mapping $\varphi_{0}: L^{1}(G, C(G, B))$ $\rightarrow\left(C(G) \rtimes_{r} G\right) \otimes t(B)$ by setting

$$
\varphi_{0}(f)\left(g_{1}, g_{2}\right):=\beta_{g_{1}^{-1}} f\left(g_{1}, g_{2}\right) .
$$

The linear mapping $\varphi_{0}$ is a $*$-homomorphism when $L^{1}(G, C(G, B))$ is equipped with the convolution twisted by the $G$-action on $C(G) \otimes B$. It is straightforward to verify that $\varphi_{0}$ is bounded in $C^{*}$-norm so we can define $\varphi:(C(G) \otimes$ $B) \rtimes_{r} G \rightarrow\left(C(G) \rtimes_{r} G\right) \otimes B$ by continuity. The $*$-homomorphism $\varphi$ is an equivariant isomorphism since an inverse can be constructed by extending

$$
\varphi^{-1}(f \otimes b)\left(g_{1}, g_{2}\right):=f\left(g_{1}, g_{2}\right) \beta_{g_{1}}(b)
$$

to a $*$-homomorphism $\varphi^{-1}:\left(C(G) \rtimes_{r} G\right) \otimes t(B) \rightarrow(C(G) \otimes B) \rtimes_{r} G$. 
Theorem 3.4 (The Pimsner-Voiculescu tower). Let $G$ be a compact Hodgkin-Lie group of rank $n$ and Weyl group of order $w$. For any separable $\hat{G}-C^{*}$ algebra A there is a $K K^{\hat{G}}$-tower
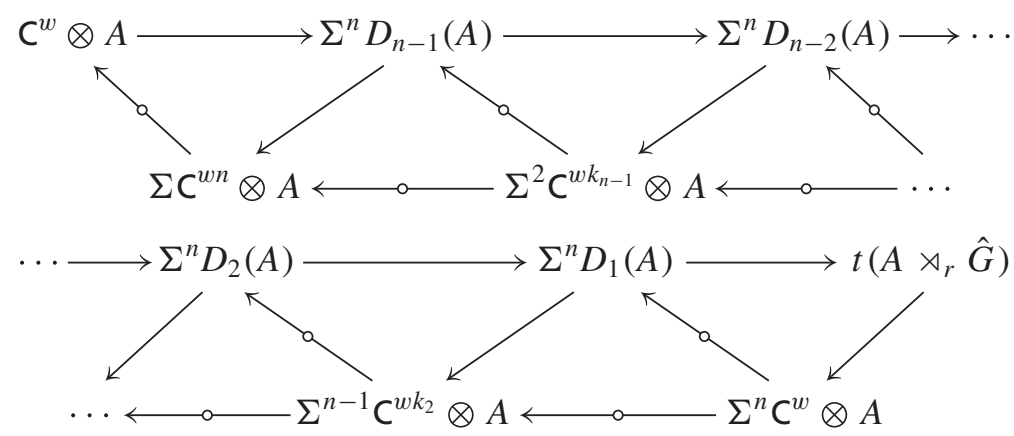

where $D_{i}(A):=\left(C_{0}\left(Y_{i}\right) \otimes \mathscr{K}\left(L^{2}(G)\right)\right) \bigotimes_{G}\left(A \rtimes_{r} \hat{G}\right)$ and is equipped with the $\hat{G}$-action induced from the diagonal $\hat{G}$-action on $C_{0}\left(Y_{i}\right) \otimes \mathscr{K}\left(L^{2}(G)\right)$.

Observe that the $D(G)$-actions on the $C^{*}$-algebras $C_{0}\left(Y_{i}\right) \otimes \mathscr{K}\left(L^{2}(G)\right)$ is defined to come from those on their Baaj-Skandalis duals $C_{0}\left(Y_{i}\right) \rtimes_{r} \hat{G}$, which are $D(G)-C^{*}$-algebras in the dual $G$-actions on the crossed products and the trivial $\hat{G}$-actions. So in general, $D_{i}(A)$ is not the tensor product of $C_{0}\left(Y_{i}\right) \otimes \mathscr{K}\left(L^{2}(G)\right)$ and $A \rtimes_{r} \hat{G}$.

Proof. By Lemma 3.3 the $\hat{G}-C^{*}$-algebra $A$ admits the equivariant Morita equivalence:

$$
\left(C(G) \otimes\left(A \rtimes_{r} \hat{G}\right)\right) \rtimes_{r} G \sim_{M} t\left(A \rtimes_{r} \hat{G}\right) .
$$

Furthermore, the isomorphism of equation (3) holds for braided tensor products over $G$ so while the $\hat{G}$-actions on $D_{i}=C_{0}\left(Y_{i}\right) \rtimes_{r} \hat{G}$ are trivial there are equivariant isomorphisms

$$
\begin{aligned}
\left(D_{i} \otimes\left(A \rtimes_{r} \hat{G}\right)\right) \rtimes_{r} G & \cong\left(\left(C_{0}\left(Y_{i}\right) \rtimes_{r} \hat{G}\right) \bigotimes_{G}\left(A \rtimes_{r} \hat{G}\right)\right) \rtimes_{r} G \\
& \cong\left(C_{0}\left(Y_{i}\right) \otimes \mathscr{K}\left(L^{2}(G)\right)\right) \bigotimes_{G}\left(A \rtimes_{r} \hat{G}\right) .
\end{aligned}
$$

Thus if we tensor the dual fundamental $G-\mathrm{PV}$-tower (11) by the $G-C^{*}$-algebra $A \rtimes_{r} \hat{G}$ we obtain a new $K K^{G}$-tower which becomes the Pimsner-Voiculescu tower of $A$ after applying Baaj-Skandalis duality, using the Morita equivalence (13) and the isomorphisms (14).

The Pimsner-Voiculescu tower (12) is the generalization of the resolution in (9) to compact Hodgkin-Lie groups. Applying the cohomological functor $K K(-, B)$ to the Pimsner-Voiculescu tower we obtain a similar resolution of 
$K K_{*}\left(A \rtimes_{r} \hat{G}, B\right)$ in terms of $K K_{*}(A, B)$ as in (9). Similarly, the homological functor $K K(B,-)$ applied to the Pimsner-Voiculescu tower gives a resolution of $K K(B, A \rtimes \hat{G})$ in terms of $K K(B, A)$. Observe that since $A$ has a $\hat{G}$-action, the groups $K K\left(\mathrm{C}^{w} \otimes A, B\right)$ and $K K\left(B, C^{w} \otimes A\right)$ will always have an $R(G)$ module structure and since $R(T)$ is free over $R(G)$ also an $R(T)$-module structure.

As an example of this, we will use the Pimsner-Voiculescu tower to calculate the $K$-theory of the homogeneous space $G / H$ when $H \subseteq G$ is a Lie subgroup. More generally, this technique can be used to calculate $K_{*}\left(A \rtimes_{r} \hat{G}\right)$ for any $\hat{G}-$ $C^{*}$-algebra $A$ when one knows $K_{*}(A)$ and its $R(G)$-module structure coming from the Julg isomorphism $K_{*}(A) \cong K_{*}^{G}\left(A \rtimes_{r} \hat{G}\right)$. To calculate $K^{*}(G / H)$, consider the $C^{*}$-algebra $A:=C^{*}(H)$ equipped with the $\hat{G}$-action induced from the natural mapping $C^{*}(H) \rightarrow \mathcal{M}\left(C^{*}(G)\right)$. Green's imprimitivity theorem implies that $C^{*}(H) \rtimes \hat{G}$ is $K K$-equivalent with $C(G / H)$. Thus, if we take the $K$-theory of the Pimsner-Voiculescu tower of $C^{*}(H)$ we obtain a tower of abelian groups of the form

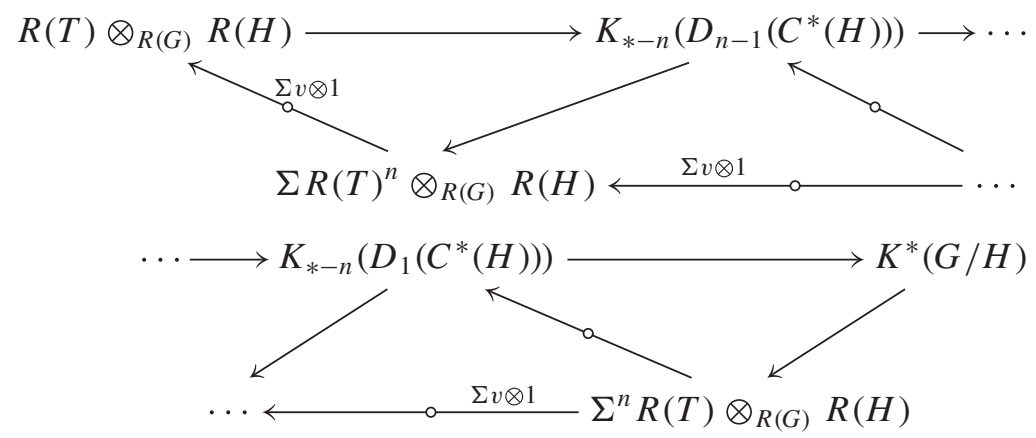

We use $\Sigma$ to denote degree shift in the category of $\mathbf{Z} / 2 \mathrm{Z}$-graded abelian groups. Here we have used that $R(T)$ is a free $R(G)$-module of rank $w$ so $K_{*}\left(C^{w} \otimes C^{*}(H)\right) \cong R(T) \otimes_{R(G)} R(H)$. Thus the lowest row is the tensor product of $R(H)$ with the Koszul complex of $R(T)$ that is associated with the regular sequence $1-t_{1}, 1-t_{2}, \ldots, 1-t_{n}$ under the isomorphism $R(T) \cong$ $\mathrm{Z}\left[t_{1}^{ \pm 1}, t_{2}^{ \pm 1}, \ldots, t_{n}^{ \pm 1}\right]$.

If we restrict our attention to simple compact Lie groups we can perform an explicit calculation of all the groups in (15). Assume that $G=G_{n}$ is a simple compact Hodgkin-Lie group in the classical $A, B, C$ - or $D$-series of rank $n$ and assume that $H=G_{k} \subseteq G_{n}$ is a simple simply connected compact Lie group in the same classical serie being of rank $k<n$. We may take a maximal torus $T_{n} \subseteq G_{n}$ such that $T_{k}:=T_{n} \cap G_{k}$ is a maximal torus in $G_{k}$. In this case we may consider $R\left(T_{k}\right)$ as an ideal in $R\left(T_{n}\right)$ and $R\left(T_{n}\right) \otimes_{R\left(G_{n}\right)} R\left(G_{k}\right) \cong R\left(T_{k}\right)$ as $R\left(T_{n}\right)$-modules. Under the isomorphisms $R\left(T_{k}\right) \cong \mathrm{Z}\left[t_{1}^{ \pm 1}, t_{2}^{ \pm 1}, \ldots, t_{k}^{ \pm 1}\right]$ and 
$R\left(T_{n}\right) \cong \mathrm{Z}\left[t_{1}^{ \pm 1}, t_{2}^{ \pm 1}, \ldots, t_{n}^{ \pm 1}\right]$, the Koszul vector $v$ is identified with $\sum_{i=1}^{k}(1-$ $\left.t_{i}\right) \mathrm{e}_{i}^{*} \in \operatorname{Hom}\left(R\left(T_{k}\right)^{n}, R\left(T_{k}\right)\right)$. Thus we arrive at the tower

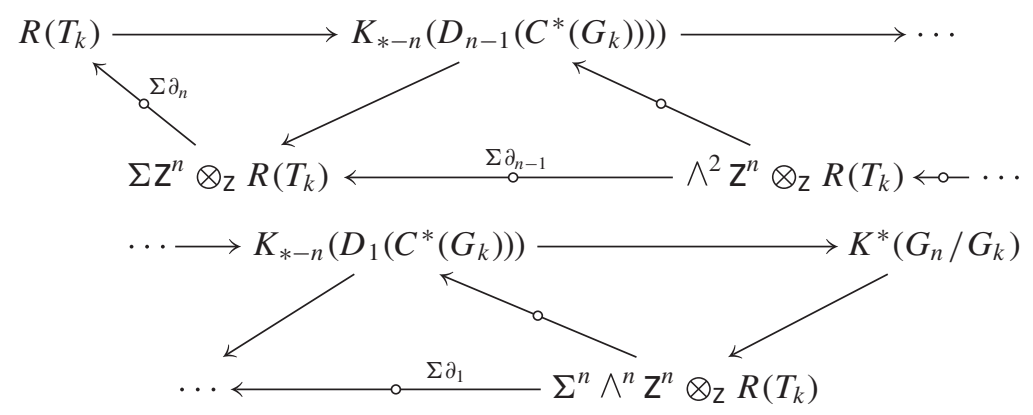

Let us use the notation $E^{*}$ for the complex $\wedge^{n-*} Z^{n} \otimes R\left(T_{k}\right)$ equipped with the Koszul differential from the vector $\sum_{i=1}^{k}\left(1-t_{i}\right) \mathrm{e}_{i}^{*}$ which we as above denote by $\partial_{l}: E^{l-1} \rightarrow E^{l}$. After some simpler calculations in this Koszul complex we arrive at the conclusion that

$$
K_{*-n}\left(D_{l}\left(C^{*}\left(G_{k}\right)\right)\right) \cong \operatorname{ker}\left(\partial_{l+1}\right) \oplus \bigoplus_{j=l+2}^{n+1} \Sigma^{n-j} H^{j}\left(E^{*}\right) .
$$

Hence we obtain the isomorphism $K^{*}\left(G_{n} / G_{k}\right) \cong \bigoplus_{j=1}^{n+1} \Sigma^{n-j} H^{j}\left(E^{*}\right)$. These cohomology groups are calculated in Corollary 17.10 of [5] and $H^{j}\left(E^{*}\right)$ is a free group of rank $k(j):=(n-k) ! /(n-j) !(n-j-k)$ ! if $0 \leq j \leq n-k$ and 0 otherwise. Therefore

$$
K^{*}\left(G_{n} / G_{k}\right) \cong \bigoplus_{j=0}^{n-k} \Sigma^{n-j} \mathrm{Z}^{k(j)}=\mathrm{Z}^{2^{n-k-1}} \oplus \Sigma \mathrm{Z}^{2^{n-k-1}} .
$$

Theorem 3.5 (The dual Pimsner-Voiculescu tower). Under the assumptions of Theorem 3.4 there is a $K K^{G}$-tower
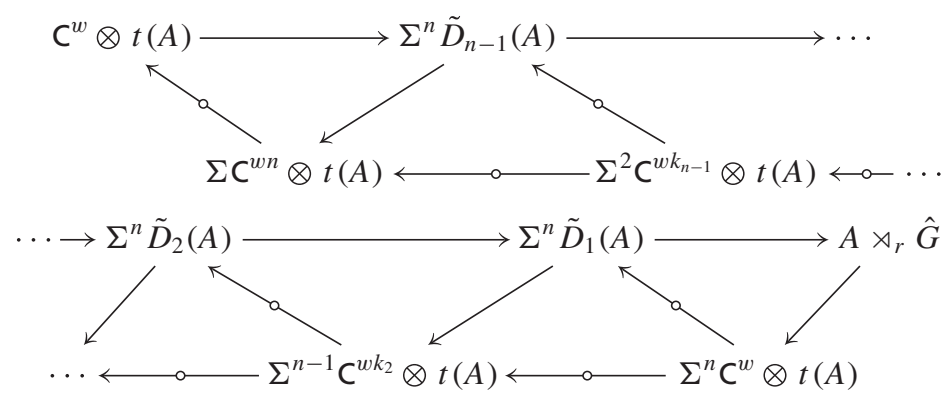

where $\tilde{D}_{i}(A):=D_{i} \otimes_{\hat{G}} A$. 
For a homological functor $F: K K^{\hat{G}} \rightarrow A b$, the dual Pimsner-Voiculescu tower of $A$ allows us to calculate $F(A)$ in terms of the objects $F\left(C_{r}^{*}(G) \otimes t(A)\right)$. As we shall see below, $\hat{G}-C^{*}$-algebras of the form $C_{r}^{*}(G) \otimes t(A)$ behaves similarly to proper actions. Compare this result to Theorem 4.4 of [8].

Proof. Consider the braided tensor product by $\Sigma^{n} A$ and the tower (10):

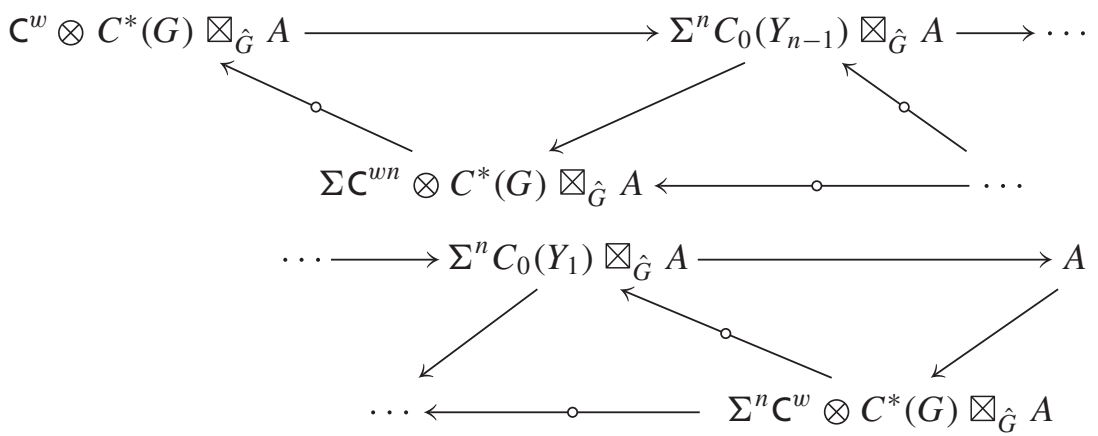

Taking crossed product between this tower and $\hat{G}$ implies the Theorem since the following equivariant Morita equivalences follows from (3)

$$
\left(C^{*}(G) \bigotimes_{\hat{G}} A\right) \rtimes_{r} \hat{G} \sim_{M} t(A)
$$

and

$$
\left(C_{0}\left(Y_{i}\right) \bigotimes_{\hat{G}} A\right) \rtimes_{r} \hat{G} \sim_{M}\left(C_{0}\left(Y_{i}\right) \rtimes_{r} \hat{G}\right) \bigotimes_{\hat{G}} A=D_{i} \bigotimes_{\hat{G}} A .
$$

One of the main motivations behind this paper was to give a precise description of the Baum-Connes property of duals of Hodgkin-Lie groups. The Baum-Connes property for coactions of compact Lie groups was given meaning to and was proved to hold in [11]. More generally, this fits into the program of generalizing the Baum-Connes property to quantum groups. So far, it is not known what a suitable property the Baum-Connes property should be for a general locally compact quantum group. For discrete quantum groups which are torsion-free, in the sense of [9], there is a formulation and as mentioned above duals of compact Hodgkin-Lie groups are torsion-free.

The problem that arises when one tries to define the Baum-Connes assembly mapping for a quantum group is that there is no natural notion of a proper action and there are in general too many quantum homogeneous spaces. It is much easier to generalize certain notions of free actions than proper actions of a quantum group by just saying that an action of a discrete quantum group $\Gamma$ on a $C^{*}$-algebra $A$ is truly free if there is a $C^{*}$-algebra $A_{0}$ and an equivariant 
*-isomorphism $A \cong A_{0} \otimes_{\min } C_{0}(\Gamma)$ with $\Gamma$ only acting on the second leg. In the case of a group, there are many free actions that are not truly free but this stronger notion of a free action will suffice for our purposes.

Restricting one's attention to generalizing the Baum-Connes property of the simpler class of torsion-free discrete groups to the quantum setting, when proper actions are free, Meyer introduced a class of quantum groups known as torsion-free in [9]. Following [9], we say that a discrete quantum group $\Gamma$ is torsion-free if every coaction of the compact quantum group $\hat{\Gamma}$ on a finitedimensional $C^{*}$-algebra is Morita equivalent to a trivial coaction on a direct sum of C:s. This fact implies that any finite-dimensional projective representation of the dual compact quantum group is equivalent to a representation. If $\Gamma$ is a discrete group, coactions of the dual compact quantum group on finitedimensional $C^{*}$-algebras that are not Morita equivalent to a trivial coaction on a direct sum of C:s correspond to finite subgroups so a discrete group is torsion-free if and only if it is torsion-free in the sense of [9].

For a torsion-free quantum group a proper action should correspond to a free action. Under Baaj-Skandalis duality, a truly free $\Gamma-C^{*}$-algebra corresponds to a trivial $\hat{\Gamma}$-action. Let $\mathscr{C} \mathscr{I}_{\hat{\Gamma}}$ denote the image of $t: K K \rightarrow K K^{\hat{\Gamma}}$. The triangulated category $\left\langle\mathscr{C} \mathscr{I}_{\hat{\Gamma}}\right\rangle$ is defined as the localizing subcategory generated by $\mathscr{C} \mathscr{I}_{\hat{\Gamma}}$. Following the formulation of [9], $\Gamma$ is said to satisfy the strong BaumConnes property if the embedding of triangulated categories $\left\langle\mathscr{C}_{\hat{\Gamma}}\right\rangle \rightarrow K K^{\hat{\Gamma}}$ is essentially surjective. The strong Baum-Connes property of $\Gamma$ is equivalent to that any $\Gamma-C^{*}$-algebra is in the localizing category generated by all truly free actions. So regardless of what notion of a proper action we choose, the strong Baum-Connes conjecture will imply that the localizing category generated by all such proper actions will be $K K^{\Gamma}$. The quantum group is said to satisfy the Baum-Connes property if the same statement holds after localizing with respect to the kernel of equivariant $K$-theory.

In [11] the Baum-Connes property was formulated in the slightly more general setting of duals of compact Lie groups. The finite-dimensional projective representations of a compact Lie group $G$ correspond to the torsion classes of $H^{2}\left(G, S^{1}\right)$, which can be thought of as the torsion of $\hat{G}$. When $G$ is Hodgkin, $H^{2}\left(G, S^{1}\right)$ is torsion-free so $\hat{G}$ is torsion-free. In this case a "proper" action is an object of the additive category generated by $\hat{G}$-algebras that are Baaj-Skandalis dual to $A_{0} \otimes \mathrm{C}_{\omega}$, with $\mathrm{C}_{\omega}$ denoting the endomorphisms of a projective representation $\omega$ and $A_{0}$ having trivial $G$-action. So the substitute in the setting of [11] for proper actions is the category of tensor products between Baaj-Skandalis duals of coactions on finite-dimensional $C^{*}$-algebras and trivial actions, just as the truly free actions form a substitute for proper actions of torsion-free quantum groups. The Baum-Connes property of coactions of a compact Hodgkin-Lie group is a direct consequence of Theorem 3.5. 
The method of proof of Proposition 2.1 of [11] can be used to generalize both Theorem 3.4 and Theorem 3.5 to arbitrary compact Lie group.

Finally, let us mention a promising generalization of Theorem 3.5 to Woronowicz deformations. It was proved in [12] that the compact quantum group $S U_{q}(2)$ satisfies that $C\left(S U_{q}(2) / T\right)$ is $K K^{D\left(S U_{q}(2)\right)}$-isomorphic to $C^{2}$ for $q \in$ ]0, 1[. So if we apply the induction functor $\operatorname{Ind}_{T}^{S U_{q}(2)}: K K^{T} \rightarrow K K^{S U_{q}(2)}$ to the distinguished triangle Baaj-Skandalis dual to (5) and use the isomorphism of Nest-Voigt we arrive at the distinguished triangle in $K K^{D\left(S U_{q}(2)\right)}$ :

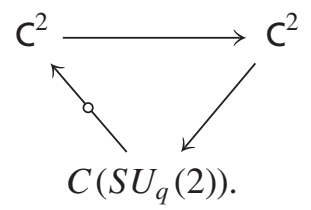

Using the technique from the proof of Theorem 3.5 any $A \in K K^{S \widehat{U_{q}(2)}}$ fits into a distinguished triangle

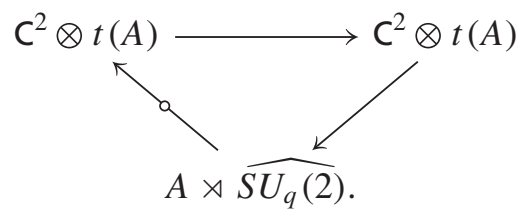

This distinguished triangle gives an alternative proof of the strong Baum-

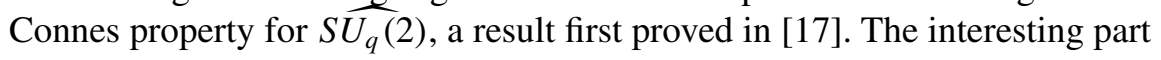
about this proof is that it only relies on the isomorphism $C\left(G_{q} / T\right) \cong \mathrm{C}^{w}$ in $K K^{D\left(G_{q}\right)}$. So if such an isomorphism exists for a simply connected semisimple compact Lie group $G$, the strong Baum-Connes conjecture holds for $\hat{G}_{q}$, the quantum dual of the Woronowicz deformation of $G$. To formulate the Baum-Connes property for $\hat{G}_{q}$ we must of course know that it is torsion-free, a statement proved in [17] for $G=S U(2)$ and the general case was proved in [6]. Another striking application of such an isomorphism is that the method above for calculating $K$-theory of homogeneous spaces can be generalized to classical quantum homogeneous spaces of the Woronowicz deformations.

\section{REFERENCES}

1. Baaj, S., and Skandalis, G., $C^{*}$-algèbres de Hopf et théorie de Kasparov équivariante, $K$ theory 2 (1989), 683-721.

2. Baaj, S., and Skandalis, G., Unitaires multiplicatifs et dualité pour les produits croisés de $C^{*}$-algèbres, Ann. Sci. École Norm. Sup. (4) 26 (1993), 425-488. 
3. Baaj, S., Vaes, S., Double crossed products of locally compact quantum groups, J. Inst. Math. Jussieu 4 (2005), 135-173.

4. Baum, P., Connes, A., and Higson, N., Classifying space for proper actions and K-theory of group $C^{*}$-algebras, pp. 240-291 in: $C^{*}$-algebras: 1943-1993, Proc. San Antonio 1993, Contemp. Math. 167, Amer. Math. Soc., Providence, RI 1994.

5. Eisenbud, D., Commutative Algebra. With a View toward Algebraic Geometry, Graduate Texts in Math. 150, Springer, New York 1995.

6. Goffeng, M., A remark on twists and the notion of torsion-free discrete quantum groups, arXiv:1004.0072, to appear in Algebr. Represent. Theory, DOI: 10.1007/s10468-0119266-6.

7. Kustermans, J., and Vaes, S., Locally compact quantum groups, Ann. Sci. École Norm. Sup. (4) 33 (2000), 837-934.

8. Meyer, R., and Nest, R., Homological algebra in bivariant K-theory and other triangulated categories I, pp. 236-289 in: Triangulated Categories, London Math. Soc. Lecture Note 375, Cambridge Univ. Press, Cambridge 2010.

9. Meyer, R., Homological algebra in bivariant K-theory and other triangulated categories II, Tbil. Math. J. 1 (2008), 165-210.

10. Meyer, R., and Nest, R., The Baum-Connes conjecture via localisation of categories, Topology 45 (2006), 209-259.

11. Meyer, R., and Nest, R., An analogue of the Baum-Connes isomorphism for coactions of compact groups, Math. Scand. 100 (2007), 301-316.

12. Nest, R., and Voigt, C., Equivariant Poincaré duality for quantum group actions, J. Funct. Anal. 258 (2010), 1466-1503.

13. Raeburn, I., On crossed products by coactions and their representation theory, Proc. London Math. Soc. (3) 64 (1992), 625-652.

14. Rosenberg, J., and Schochet, C., The Künneth theorem and the universal coefficient theorem for equivariant $K$-theory and $K K$-theory, Mem. Amer. Math. Soc. 62:348 (1986).

15. Steinberg, R., On a theorem of Pittie, Topology 14 (1975), 173-177.

16. Vaes, S., A new approach to induction and imprimitivity results, J. Funct. Anal. 229 (2005), 317-374.

17. C. Voigt, The Baum-Connes conjecture for free orthogonal quantum groups, Adv. Math. 227 (2011), 1873-1913.

MATHEMATICAL SCIENCES

CHALMERS UNIVERSITY OF TECHNOLOGY AND THE UNIVERSITY OF GOTHENBURG

SE-412 96 GÖTEBORG

SWEDEN

E-mail: goffeng@chalmers.se 